\title{
Optical Model And Cross Section Uncertainties
}

\author{
M. Herman ${ }^{1, a}$, M.T. Pigni ${ }^{1}$, F.S. Dietrich ${ }^{2}$, and P. Obložinský ${ }^{1}$ \\ 1 National Nuclear Data Center, Brookhaven National Laboratory, Upton, NY 11973-5000, U.S.A. \\ 2 Nuclear Theory \& Modeling Group, Lawrence Livermore National Laboratory, Livermore, CA 94551, U.S.A.
}

\begin{abstract}
Distinct minima and maxima in the neutron total cross section uncertainties were observed in model calculations using spherical optical potential. We found this oscillating structure to be a general feature of quantum mechanical wave scattering. Specifically, we analyzed neutron interaction with ${ }^{56} \mathrm{Fe}$ from $1 \mathrm{keV}$ up to 65 $\mathrm{MeV}$, and investigated physical origin of the minima. We discuss their potential importance for practical applications as well as the implications for the uncertainties in total and absorption cross sections.
\end{abstract}

\section{Introduction}

Strongly fluctuating structures were observed in the modelcalculated uncertainties of nuclear reaction cross sections reported in [1]. The effect is particularly pronounced in the total and elastic scattering leading to the distinct minima in the uncertainties, which imply that the precision of the model calculations at these specific energies is extraordinary high. Fig. 1 shows the above mentioned structure in the case of neutron total cross sections on ${ }^{127} \mathrm{I}$. One notes that the uncertainty correlation matrix resembles 'chess board' with fully correlated regions bordering fully anti-correlatd ones. Energies of these borders coincide with the uncertainty minima observed in the cross sections. Very similar pattern has also been observed in the elastic scattering.
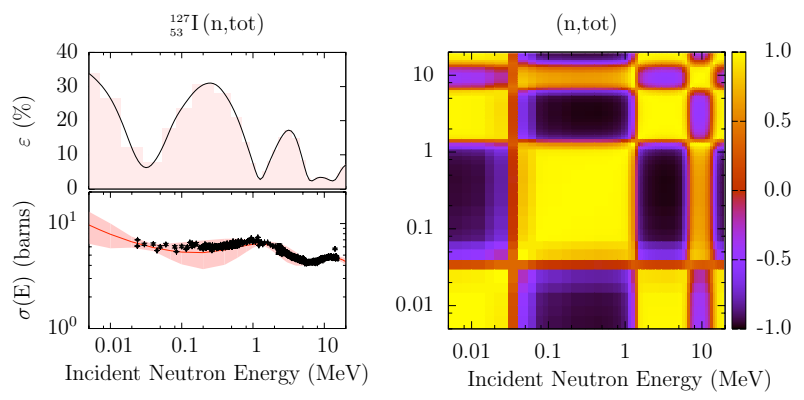

Fig. 1. Uncertainties (left panel) and their correlations (right panel) for the ${ }^{127} \mathrm{I}+\mathrm{n}$ total cross sections obtained by perturbing depth of the real potential (the perturbation was exaggerated to enhance the visibility of the fluctuating structure). Optical model calculated cross sections and experimental data are drawn together with the uncertainties (shaded area) in the lower part of the left panel.

\footnotetext{
a e-mail: mwherman@abnl.gov
}

The results reported in [1] indicate that the oscillating structure in the uncertainties of the total and elastic cross sections is common for many nuclei. Fig. 2 summarizes these results showing that the minima form intriguing regular patterns when plotted in the energy-mass plane.

Taking in to account that the total cross sections are fully determined by the optical model the physical origin of the uncertainty minima must be traceable to the OM physics. The optical model is a critically important ingredient in nuclear reaction calculations, since it yields the cross section for compound nuclear formation in the initial stage of a reaction, and supplies the transmission coefficients for branching into the various final states. Therefore, the uncertainty minima have potentially far reaching consequences on the precision of the nuclear data through determination of the optical model (OM) parameters and the way the experiments are planned. In this contribution, we investigate physics origin of the uncertainty minima and argue that they are the universal feature of quantum wave scattering. Finally, we discuss practical implications of this finding.

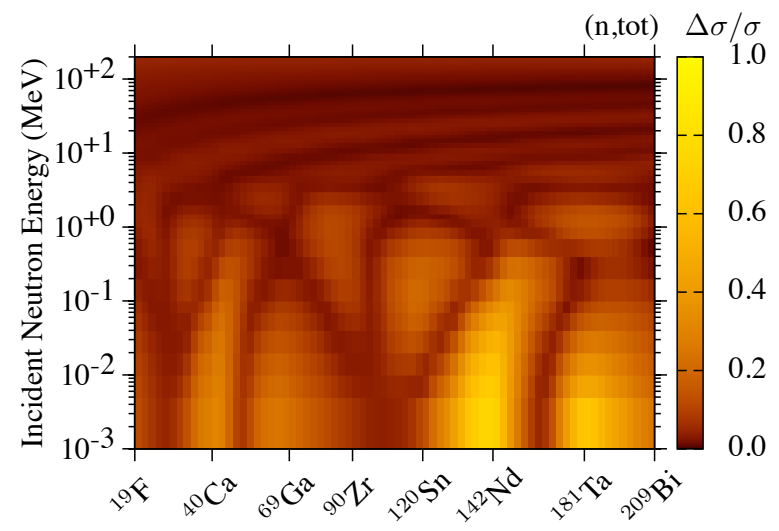

Fig. 2. Relative uncertainties for neutron total cross sections obtained in Ref. [1] using spherical optical potential [2].

This is an Open Access article distributed under the terms of the Creative Commons Attribution-Noncommercial License, which permits unrestricted use, distribution, and reproduction in any noncommercial medium, provided the original work is properly cited. 


\section{Calculations}

The present study was carried out for nuclei across the periodic table $\left({ }^{19} \mathrm{~F}\right.$ to $\left.{ }^{209} \mathrm{Bi}\right)$, and for a broad range of neutron energies (up to $200 \mathrm{MeV}$ ). We used global parameterization of the spherical OM by Koning-Delaroche [2]. We chose spherical OM in order to avoid coupled-channels effects that would obscure clean picture of wave scattering on a spherical nucleus, understanding that for the deformed nuclei our results are only conceptual.

We investigated the sensitivity of the total $\sigma_{\text {tot }}$ and absorption $\sigma_{\mathrm{abs}}$ cross sections to the variation of 15 optical model parameters including strength, radius, and diffuseness of the optical potential. Our definition of sensitivity reads

$$
\mathcal{D}(E ; p)=\frac{\Delta p}{p} \frac{\partial \sigma}{\partial p} \frac{p}{\sigma}=\frac{\Delta \sigma}{\sigma} .
$$

Here, $\Delta p$ represents the plausible perturbation of the parameter $p$ and $\Delta \sigma$ the change in $\sigma$ produced by $p+\Delta p$.

As an example, we consider interaction of a neutron with ${ }^{56} \mathrm{Fe}$. In Fig. 3 we show the response of $\sigma_{\text {tot }}$ and $\sigma_{\text {abs }}$ to the variation of 9 parameters of the OM [2]. We neglected the spin-orbit parameters because of their negligible effect.

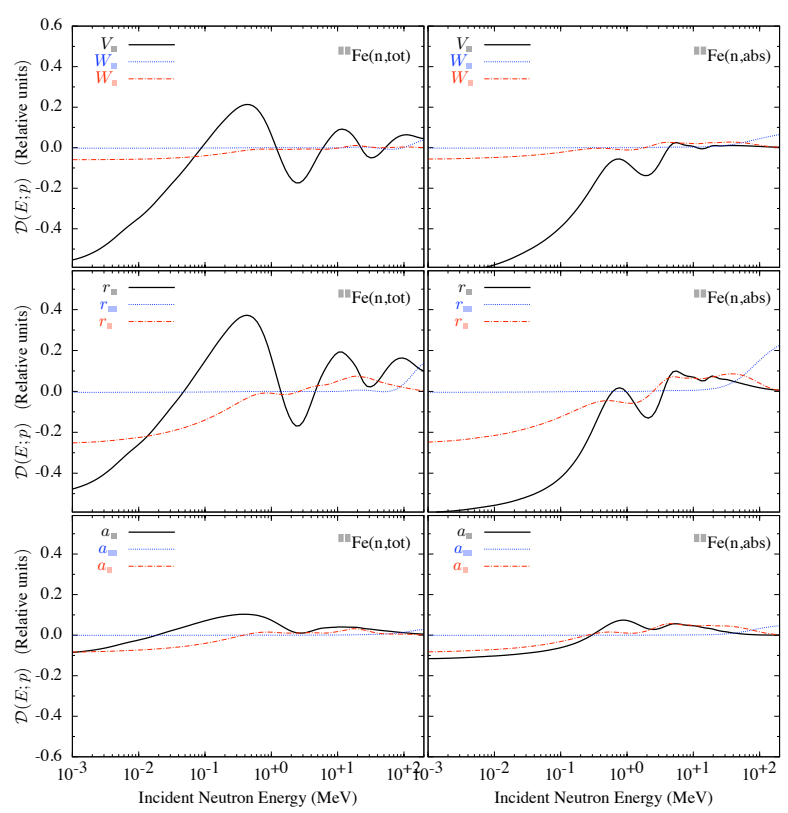

Fig. 3. Relative sensitivity of the ${ }^{56} \mathrm{Fe}+\mathrm{n}$ total and absorption cross sections to $\pm 5 \%$ perturbation of energy-dependent well depths, nuclear radii, and diffuseness.

Some of the sensitivities (e.g., $r_{v}$ and $V_{v}$ ) change sign several times between $1 \mathrm{keV}$ and $200 \mathrm{MeV}$. The immediate consequence of this behavior is that at the zero-crossing points the parameter uncertainties (even if arbitrarily large) do not contribute to the uncertainty of the cross section. We note, that only $r_{w}, W_{v}$ and $r_{s}, W_{s}$, show significant sensitivities. It is remarkable that at low energies sensitivity to the real volume parameters is larger than the sensitivity to the imaginary surface part.

\section{Origin of the Uncertainty Minima}

The relative sensitivity defined by Eq. (1) can be split into partial wave sensitivities,

$$
\mathcal{D}(E ; p)=\frac{\pi \lambda}{\sigma} \sum_{l j} \tilde{\mathcal{D}}_{l j}(E ; p),
$$

Fig. 4 shows the effect of $\mathcal{D}_{l j}\left(E ; V_{v}\right)$ on ${ }^{56} \mathrm{Fe}$ (n,tot) for different partial waves. We choose to discus sensitivity to $V_{v}$ because of its major contribution. The role of the real potential radius $r_{v}$ is actually even bigger but strong correlation between $V_{v}$ and $r_{v}$ (see Fig. 3) allows to restrict the discussion to only one of them.

As expected, the first minimum around $0.1 \mathrm{MeV}$ is entirely due to the s-wave, which dominates in this low energy range. The s-wave sensitivity changes sign and crosses zero close to $0.1 \mathrm{MeV}$ causing the first minimum in the cross section. At energies above $1 \mathrm{MeV}$, other partial waves come into play. The minimum at $1.1 \mathrm{MeV}$ results from the interplay among s-, p-, and d-waves. Actually, change in the s-wave happens to be counterbalanced by changes in the $\mathrm{p}$ - and d-waves leading to the vanishing total sensitivity. We recall that, generally, vanishing sensitivity to the major parameter is producing a minimum in the cross section uncertainties, while change in the sign of the sensitivity tends to revert the sign of the corresponding correlation matrix element.

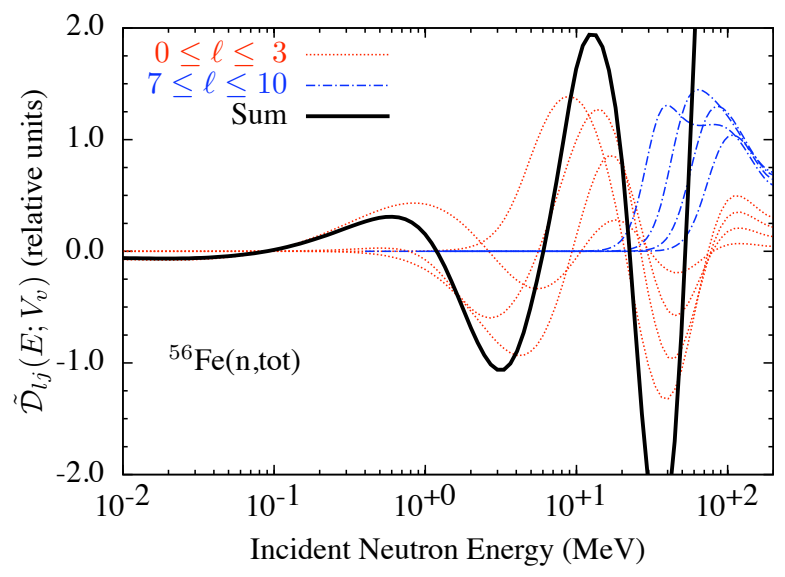

Fig. 4. Relative sensitivity of the ${ }^{56} \mathrm{Fe}+\mathrm{n}$ total cross section to $\pm 5 \%$ perturbation of optical parameters, $V_{v}$. Also shown is the effect of this perturbation on the partial wave sensitivities.

Similar considerations can be extended to the third minimum at $5 \mathrm{MeV}$ where positive sensitivity in the f-wave is annihilated by the negative sensitivity in s- and p-wave. This minimum can also be interpreted, however, in terms of the single-phase model, or nuclear Ramsauer effect [3, 4].

The Ramsauer model is a semi-classical approach in which all partial waves are assumed to have the same phase shift. The model yields an effective $S$-matrix,

$$
S_{e f f}(E)=e^{2 i \delta_{e f f}(E)}=\alpha(E) e^{i \beta(E)},
$$


where $0<\alpha<1$ generally reflects both the absorption and the averaging of various phase shifts, and $\beta$ represents the relative phase between the wave that passes through the nucleus and the waves that go around. Therefore, the total cross section is reduced to

$$
\sigma_{\text {tot }}=2 \pi(R+\lambda)^{2}\left(1-\operatorname{Re} S_{e f f}\right),
$$

where $R=r_{0} A^{1 / 3}$ is the nuclear radius and $\lambda$ is the reduced wavelength of the neutron. We have used Ramsauer model

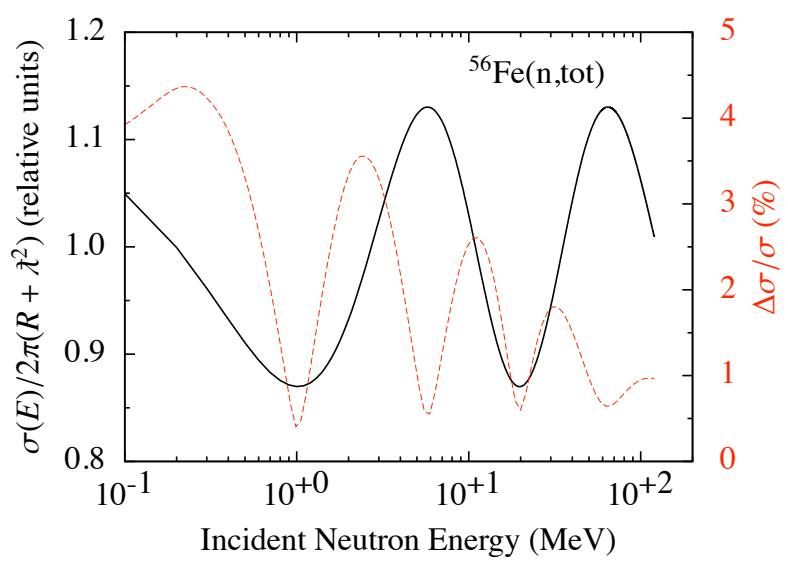

Fig. 5. Neutron total cross sections $\sigma_{\text {tot }}$ (black line) of ${ }^{56} \mathrm{Fe}$ defined in Eq. (4). Also show (in red) the relative cross section uncertainties obtained by perturbating the parameters $a, b, c, k^{\prime}$, and $r_{0}$ in Eq. (5).

to calculate ${ }^{56} \mathrm{Fe}+\mathrm{n}$ total cross sections and related uncertainties adopting the parametrization suggested in [4]. In a non-relativistic approximation, the phase angle $\beta$ in Eq. (3) takes the form

$$
\frac{\beta}{A^{1 / 3}}=c \cdot\left\{[\sqrt{a+b E}-\sqrt{E}]+k^{\prime}[\sqrt{a+b E}-\sqrt{E}]^{2}\right\},
$$

where the nuclear radius $R$ has been explicitly included in the parameter $c$ and the quantity $a$ corresponds to the depth of the real optical potential $V_{v}$. Following [4] we assume $a=35.0 \pm 0.5 \mathrm{MeV}, b=0.80 \pm 0.01, c=0.46 \pm 0.01$, and $k^{\prime}=0.07 \pm 0.002$. The amplitude $\alpha=0.18-0.013 A^{1 / 3}$ was used in Eq. 4. Fig. 5 shows the results of these calculations including the uncertainties. It is remarkable that the simplified Ramsauer model predicts minima at about 1 , 5, 20 and $65 \mathrm{MeV}$ very close to the more detailed optical model calculations. This agreement justifies interpretation of the high energy minima, involving large number of partial waves, it terms of the single phase Ramsauer model.

Finally, we studied analytical solution of a plane wave scattering from the rectangular potential well. The derivative of the calculated total cross section with respect to the well depth, which directly defines uncertainty, exhibits oscillating structure similar to the one observed in the OM calculations. As expected, positions of the minima do not coincide with those deduced from the OM but the essential similarity is evident.
Summarizing these finding we retain that the minima are a natural consequence of the quantum mechanical scattering and their presence is universal. A natural question to be asked next is whether the minima are stable with respect to the choice of the optical potential?

\section{Stability of the Uncertainty Minima}

Practical importance of the discussed minima would increase enormously if they were independent from the $\mathrm{OM}$ potential used in the calculations. In order to study this facet we calculated cross section uncertainties of ${ }^{56} \mathrm{Fe}(\mathrm{n}, \mathrm{tot})$ for a few commonly used global OM potentials. Fig. 6 shows that the oscillatory behavior of the uncertainties is observed for all considered potentials. While values of the uncertainties differ considerably among the potentials, the positions of the minima agree almost perfectly. It is remarkable that the values of the uncertainties tend to agree at the minima.

Fig. 7 shows that the above findings can be extended for other nuclei and are valid also for the absorption cross section. Calculations were performed for 75 nuclei along the $\beta$-stability line for three global OM potentials (KoningDelaroche, Walter-Guss, and Becchetti-Greenlees). The characteristic patterns are quite similar for the three potentials. Below $1 \mathrm{MeV}$ patterns drawn by the uncertainty minima of total and absorption resemble each other. Above $1 \mathrm{MeV}$ the structure in total is utterly different from the one observed in the absorption. The parallel valleys predicted by the Ramsauer model, which dominate the total, are absent in the absorption. In general, the uncertainty minima in the total and in the absorption do not coincide.

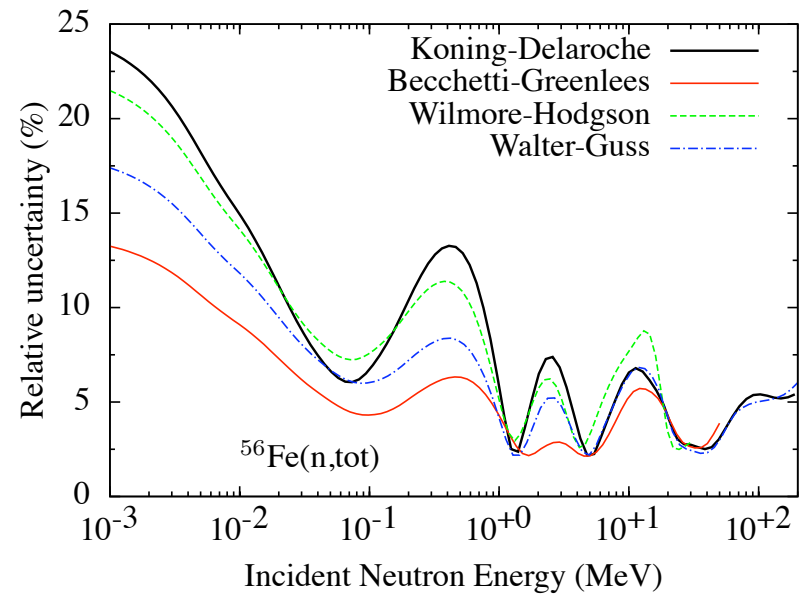

Fig. 6. Cross section uncertainties of ${ }^{56} \mathrm{Fe}+\mathrm{n}$ obtained by perturbing the optical model parameters for different global potentials [2, 5-7]. 


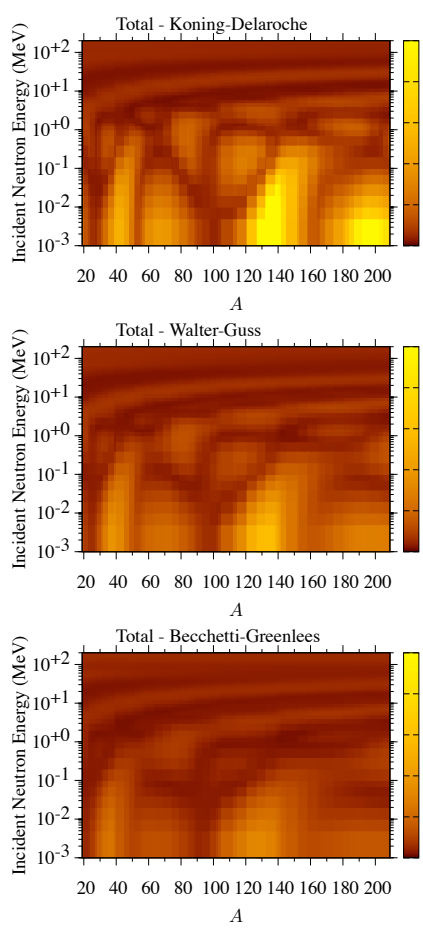

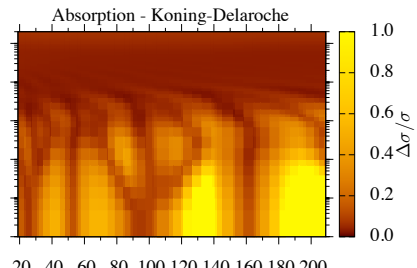

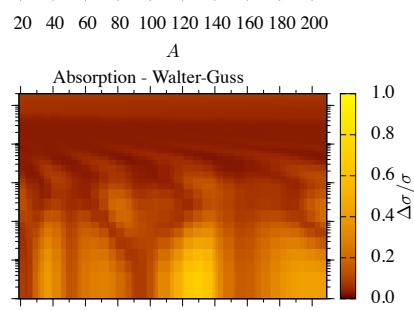

$2040 \quad 6080100120140160180200$ A Absorption - Becchetti-Greenlees

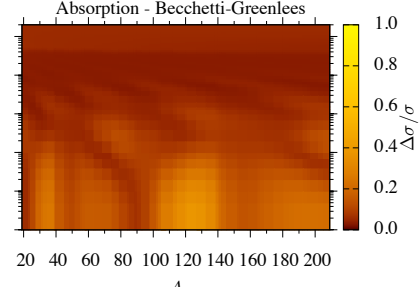

Fig. 7. Comparison of relative uncertainties obtained with three parameterizations of spherical OM potentials. Shown are uncertainties of the total and absorption cross sections for neutrons interacting with 75 materials.

\section{Conclusions}

We find that the uncertainty minima are inherent feature of the quantum mechanical wave scattering and their detailed origin depends on the incident energy. The first minimum at $0.1 \mathrm{MeV}$, is exclusively due to the zero crossing of the s-wave sensitivity, while the minima up to $5 \mathrm{MeV}$ appear to be the result of the cancelation of the sensitivities among different partial waves. The two remaining minima can be explained in terms of the Ramsauer model, predicting single-phase for all scattering waves.

The minima were found to be stable, i.e., independent from the OM potential and universal, i.e., are being observed in all nuclei. The minima are associated with the characteristic 'chess-board' like structure in the correlation matrix.

The uncertainty minima might, in principle, influence precision of the nuclear data and affect nuclear applications. In the narrow energy regions of the minima the total and absorption cross sections can be predicted with higher precision and reliability. Such regions would be perfect for defining standards and for calibrating experimental setups or normalizing relative measurements. Precise experiments carried out in the minima could be used to investigate intrinsic model deficiencies since effect of the uncertainties on the model parameters would be largely suppressed. On the other hand, the experimentalist should avoid these energy ranges when deriving parameters of the optical potential. On the contrary, the regions of high sensitivity between the minima are particularly suited for the determination of OM parameters.
More detailed studies, taking into account nuclear deformation and using coupled channels approach are needed to affirm our conclusions on a global scale. It is expected that uncertainty of the nuclear deformation and ambiguity of the collective level coupling may weaken present conclusions in case of the deformed nuclei.

\section{References}

1. M.T. Pigni, M. Herman, P. Obložinský, Extensive set of cross section covariance estimates in the fast neutron region, Nucl. Sci. Eng. 162, 25 (2009).

2. A.J. Koning and J.P. Delaroche, Local and Global Nucleon Optical Model from $1 \mathrm{keV}$ to $200 \mathrm{MeV}$, Nucl. Phys. A, 713, 231 (2003).

3. S.M. Grimes, J.D. Anderson, R.W. Bauer, et al., Justification of a Simple Ramsauer Model for Neutron Total Cross Sections, Nucl. Sci. Eng., 130, 340 (1998).

4. R.W. Bauer, J.D. Anderson, S.M. Grimes, et al., Application of a Simple Ramsauer Model for Neutron Total Cross Sections, Nucl. Sci. Eng., 130, 348 (1998).

5. F. D. Becchetti, Jr., and G. W. Greenlees, "NucleonNucleus Optical-Model Parameters, A $i 40, E_{i} 50 \mathrm{MeV}$," Phys. Rev., 182, 1190 (1969).

6. D. Wilmore and P. E. Hodgson, "The Calculation of neutron cross-sections from optical potentials," Nucl. Phys., 55, 673 (1964).

7. R. L. Walter and P. P. Guss, "A global optical model for neutron scattering for $\mathrm{A}_{i} 53$ and $10 \mathrm{MeV}_{i} 80 \mathrm{MeV}$," Radiat. Eff. 95, 73 (1985). 